\title{
Electronic structure of Te- and As-covered $\mathrm{Si}(211)$
}

\author{
Prasenjit Sen, Inder P. Batra, S. Sivananthan, and C. H. Grein \\ Department of Physics, University of Illinois at Chicago, Chicago, Illinois 60607-7059, USA \\ Nibir Dhar \\ US Army Research Laboratory, Adelphi, Maryland 20783-1197, USA \\ S. Ciraci \\ Department of Physics, Bilkent University, Ankara 06533, Turkey
}

(Received 10 September 2002; revised manuscript received 14 April 2003; published 22 July 2003)

\begin{abstract}
Electronic and atomic structures of the clean and As- and Te-covered $\mathrm{Si}(211)$ surface are studied using pseudopotential density-functional method. The clean surface is found to have $(2 \times 1)$ and rebonded $(1 \times 1)$ reconstructions as stable surface structures, but no $\pi$-bonded chain reconstruction. Binding energies of As and Te adatoms at a number of symmetry sites on the ideal and $(2 \times 1)$ reconstructed surfaces have been calculated because of their importance in the epitaxial growth of CdTe and other materials on the $\operatorname{Si}(211)$ surface. The special symmetry sites on these surfaces having the highest binding energies for isolated As and Te adatoms are identified. But more significantly, several sites are found to be nearly degenerate in binding-energy values. This has important consequences for epitaxial growth processes. Optimal structures calculated for 0.5 monolayer of As and Te coverage reveal that the As adatoms dimerize on the surface while the Te adatoms do not. However, both As- and Te-covered surfaces are found to be metallic in nature.
\end{abstract}

DOI: 10.1103/PhysRevB.68.045314 PACS number(s): 68.35. $-\mathrm{p}$, 68.43. $-\mathrm{h}$, 61.66. $-\mathrm{f}$, 73.20. $-\mathrm{r}$

\section{INTRODUCTION}

Silicon surfaces have been the subject of intense theoretical and experimental investigations. However, the high index surfaces have not received nearly as much attention as the low index ones. The (001) and (111) surfaces of Si have been studied most extensively. Only recently, the (211), (311), (331), and other higher index surfaces have attracted some attention. ${ }^{1,2}$ The emerging interest in the high Millerindex surfaces is primarily due to the fact that these surfaces may play important role in technological applications. An interesting property of many of the higher index surfaces of $\mathrm{Si}$ is the occurrence of steps and terraces. The $\mathrm{Si}(211)$ surface, for example, can be looked at as a stepped arrangement of narrow (111) terraces. A top view of the ideal $\mathrm{Si}(211)$ surface is shown in Fig. 1. The surface consists of two-atom wide terraces along [111]. Two consecutive terraces are separated by steps and are 9.41- $\AA$ apart in the [111] direction, while they extend infinitely along [011] . The atoms marked $\mathrm{T}$ on the terrace are threefold coordinated and so have one dangling bond each, while those on the step edge marked $\mathrm{E}$ are twofold coordinated and have two dangling bonds each. The behavior and possible reconstructions of such stepped surfaces are of fundamental interest as they can involve physics not seen in the lower index surfaces. On the other hand, these high index surfaces can be the natural choice for epitaxial growth of polar (both III-V and II-VI) semiconductors on a $\mathrm{Si}$ substrate. As discussed ${ }^{3}$ earlier, $\mathrm{Si}(211)$ surface leads to a better quality epitaxial growth of $\mathrm{GaP}$ as compared to $\mathrm{Si}(001)$ because it satisfies both the requirements of interface neutrality and offering inequivalent binding sites to $\mathrm{Ga}$ and $\mathrm{P}$. The $\mathrm{Si}(211)$ surface has atoms with both one and two dangling bonds. The atoms with two dangling bonds can accomodate $\mathrm{P}$, whereas $\mathrm{Ga}$ binds with
$\mathrm{Si}(211)$ that has a single dangling bond. Large area high quality CdTe layers have also been grown on the $\mathrm{Si}(211)$ surface for subsequent growth of $\mathrm{HgCdTe},{ }^{4,5}$ One basic point to understand about a surface is its possible reconstruction. There have been a few studies dealing with the reconstruction of $\mathrm{Si}(211)$. However, the results have not been conclusive. Scanning tunneling microscope (STM) studies by Berghaus et al. ${ }^{6}$ reveal both regions of $(2 \times 1)$ reconstruction and missing edge atoms possibly leading to a rebonded $(1 \times 1)$ surface (discussed below). Low-energy electron diffraction (LEED) and STM studies by Wang and Weinberg ${ }^{7}$ reveal a weak $2 \times$ reconstruction along the $[01 \overline{1}]$ direction and a definite $2 \times$ reconstruction along [11̄i].$(2 \times$ reconstruction here means a doubling of the unit cell in the corresponding direction.) Olshanetsky and Mashanov observed ${ }^{2}$ a $(4 \times 2)$ structure in their LEED studies on the $\mathrm{Si}(211)$ surface. Kaplan ${ }^{8}$ observed different structures under different conditions of surface preparation. Wright et al. observed both $(1 \times 2)$ and $(4 \times 2)$ reconstructions of the clean $\mathrm{Si}(211)$ surface annealed in vacuum. ${ }^{3}$ The properties of the clean surface being not fully understood as they are, there have been few studies of adsorption of other elements on the $\mathrm{Si}(211)$ surface. Such studies are important in the context of epitaxial growth of other materials on Si. Wright et al. ${ }^{3}$ found both $(6 \times 1)$ and $(6 \times 2)$ reconstructions on $\mathrm{Ga}$ deposited $\mathrm{Si}(211)$ surface, whereas Kaplan ${ }^{8}$ found only a $(6 \times 1)$ pattern in his LEED studies of the same surface. Yang and Williams, ${ }^{9}$ in their study on the effects of carbon contamination on the $\mathrm{Si}(211)$ surface, found that while a small amount of $\mathrm{C}$ removes the $(1 \times 2)$ reconstruction of the clean surface and leads to a $(4 \times 2)$ reconstruction, larger quantities of $\mathrm{C}$ lead to facetting. Michel et al. have studied $\mathrm{Br}$ adsorption on the $\mathrm{Si}(211)$ surface $^{10}$ by an $\mathrm{x}$-ray standing-wave technique. They conclude that $\mathrm{Br}$ adsorption reverts the $(2 \times 1)$ reconstruction 

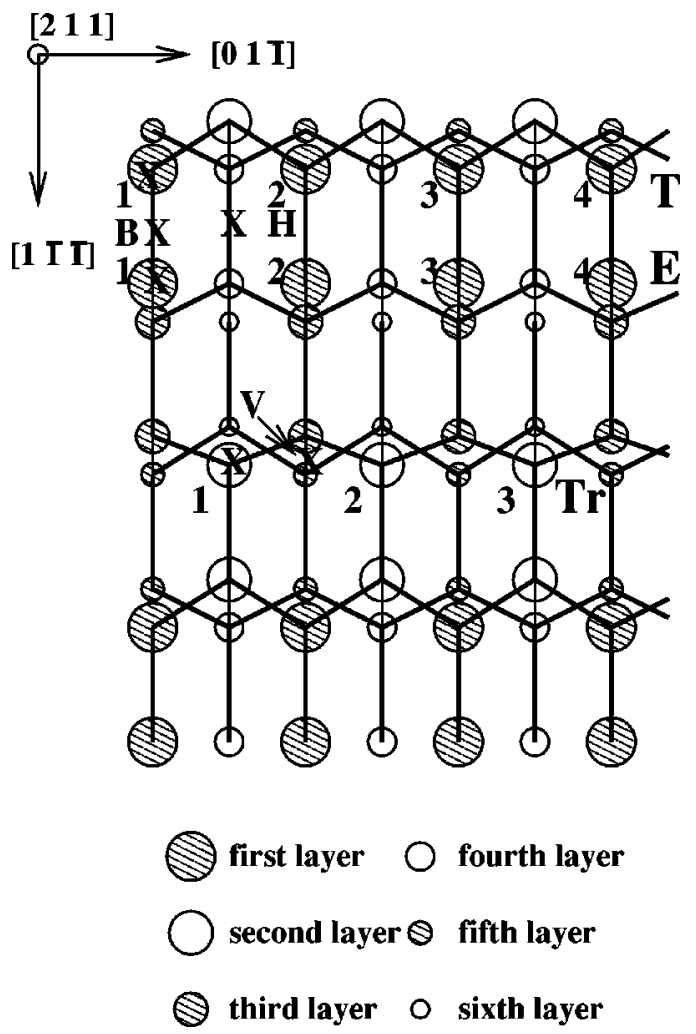

FIG. 1. Top view of an ideal bulk-terminated $\mathrm{Si}(211)$ surface. The top layer terrace and edge atoms and the second-layer trench atoms are marked by $\mathrm{T}, \mathrm{E}$, and $\mathrm{Tr}$, respectively. The other symmetry sites at which adsoprtion of As and Te is studied have also been marked (by X).

of the clean surface into $(1 \times 1)$ and that $\mathrm{Br}$ is adsorbed at least at two different sites on the surface. A similar conclusion is reached by Dhar et al. ${ }^{11}$ in their study of Te adsorption on the $\mathrm{Si}(211)$ surface. Te is found to adsorb at more than one site. This work also estimates the binding energy of Te on $\mathrm{Si}(211)$ to be $3.46 \mathrm{eV} /$ atom. There have been even fewer theoretical studies on the $\mathrm{Si}(211)$ surface. Chadi ${ }^{1}$ studied the atomic structure of the (211), (311), and (331) surfaces of $\mathrm{Si}$ by the tight-binding approach. The two firstprinciples calculations are by Grein ${ }^{12}$ and Mankefors. ${ }^{13}$ Of these, the second one studies a system with only two atoms in each (211) atomic plane (30 atomic planes containing 60 atoms). In view of the fact that each terrace contains two inequivalent atoms, this system cannot give any information about possible reconstructions, as for example, a $2 \times$ reconstruction along [01 $\overline{1}]$ would require at least two (equivalent) edge atoms. To our knowledge, there has also been no theoretical study of adsorption of any material on the $\mathrm{Si}(211)$ surface. For epitaxial growth of various materials on the $\mathrm{Si}(001)$ surface (e.g., Ge, which normally grows in the Stranski-Krastanov mode), it has been found that As or Te acts as a surfactant, leading to a nice layer-by-layer growth. It is expected that these materials can act as good surfactants even on the $\mathrm{Si}(211)$ surface. Hence, one would like to understand the energetics and structure of As and Te adsorption on $\mathrm{Si}(211)$. Moreover, the structure of a Te layer on $\mathrm{Si}(211)$ is also important in the context of CdTe growth on this surface. With these applications in mind, and also to understand its structure and electronic properties, we performed extensive first-principles calculations on the clean and As- and Te-covered $\mathrm{Si}(211)$ surface. We organize the rest of the paper in the following way. Section II gives the parameters we used in the pseudopotential density-functional calculations. Section III discusses the results of our calculation. Section III A gives the results for the clean surface while Secs. III B and III C give the results of As and Te adsorption on the ideal and $(2 \times 1)$ reconstructed surfaces. Finally, in the closing Sec. IV, we state our main conclusions.

\section{METHOD}

First-principles calculations are carried out within the density-functional theory. The $\operatorname{Si}(211)$ surface is represented in a repeated slab geometry. Each slab contains seven $\operatorname{Si}(211)$ layers and a $12-\AA$ vacuum region. Each layer contains eight $\mathrm{Si}$ atoms - two along [111] and four along [01흐. In other words, each layer contains four edge and four terrace $\mathrm{Si}$ atoms. The $\mathrm{Si}$ atoms in the bottom layer have their dangling bonds saturated by $\mathrm{H}$ atoms. Since the edge atoms have two and the terrace atoms have one dangling bond each, we require $12 \mathrm{H}$ atoms to saturate all the dangling bonds on the bottom surface. The top five Si layers are relaxed for geometry optimization while the two lowermost $\mathrm{Si}$ and $\mathrm{H}$ layers are held fixed to simulate the bulklike termination. The wave functions are expanded in a plane-wave basis set with a cutoff energy $|\vec{k}+\vec{G}|^{2} \leqslant 250 \mathrm{eV}$. We carried out some limited convergence studies, discussed in the following section, to ensure that this energy cutoff gives sufficiently converged values for the binding energies. The Brillouin-zone integration is performed within a Monkhorst-Pack (MP) scheme using four inequivalent $k$ points. As we shall see, a doubling of this mesh changes binding energies by less than $1 \mathrm{meV}$ per atom. Ionic potentials are represented by Vanderbilt-type ultra soft pseudopotentials ${ }^{14}$ and results are obtained using generalized gradient approximation ${ }^{15}$ for the exchange-correlation potential. Preconditioned conjugate gradient is used for wave-function optimization and a conjugate gradient for ionic relaxations. The $z$ axis is taken perpendicular to the $\underline{\operatorname{Si}}(211)$ surface, while $x$ and $y$ axes are along $[01 \overline{1}]$ and $[1 \overline{1} \overline{1}]$, respectively. All our calculations are performed using the VASP code. ${ }^{16}$

\section{RESULTS}

\section{A. Clean surface}

We performed a total-energy calculation using the above parameters for an ideal surface where all the Si atoms are fixed at their bulk-terminated positions (cubic lattice constant $5.43 \AA$ ). We have studied the clean surface here even though these calculations are already in print. ${ }^{12}$ The main reason being that we need information about the clean system, to arrive at binding-energy values for the adsorbates. For good accuracy, all calculations should be performed using the same set of calculational parameters. The following two tables show that the cutoff energy and $k$ mesh used in our 
TABLE I. Total binding energy $\left(E_{b}\right)$ of the clean $\mathrm{Si}(211)$ surface for different $E_{\text {cut }}$ with four irreducible $k$ points.

\begin{tabular}{lc}
\hline \hline$E_{\text {cut }}(\mathrm{eV})$ & $E_{b}(\mathrm{eV})$ \\
\hline 100 & -316.598285 \\
150 & -323.238185 \\
200 & -324.794424 \\
250 & -325.044874 \\
300 & -325.075853 \\
\hline \hline
\end{tabular}

investigations lead to well converged results. Table I gives the total binding energy of the clean surface, defined as the difference between the total energy of the system and the total energy of all its isolated constituent atoms, for different $E_{c u t}$ values for a fixed $(2 \times 2 \times 1)$ MP $k$ mesh (four irreducible $k$ points). The difference between the binding energies with $E_{\text {cut }}=250 \mathrm{eV}$ and $300 \mathrm{eV}$ is $4 \times 10^{-4} \mathrm{eV}$ per atom, which is beyond the limit of accuracy of the present calculations ( $1-2 \mathrm{meV}$ per atom). Hence we settled on the $E_{\text {cut }}$ $=250 \mathrm{eV}$. With this information in hand, we increased the $k$ mesh. In Table II, we show the total binding energy of the system for different $k$ meshes with a fixed $E_{\text {cut }}=250 \mathrm{eV}$. We find that the difference in the total binding energies with $(2 \times 2 \times 1)$ and $(4 \times 4 \times 1) k$ meshes is $8 \times 10^{-4} \mathrm{eV}$ per atom, which is again beyond the limit of accuracy for the present calculations. Hence, all subsequent calculations are performed with $E_{c u t}=250 \mathrm{eV}$ and the $k$ mesh derived from (2 $\times 2 \times 1$ ). Our computed results do not differ significantly from the previous findings ${ }^{12}$ for clean and reconstructed $\mathrm{Si}(211)$ surfaces, but are expected to be somewhat more accurate due to refined mesh and the usage of ultrasoft pseudopotentials. The presence of the two dangling bonds at each edge atom of $\mathrm{Si}(211)$ can readily lead to dimerization along the [011] direction. In the optimized geometry, the $\mathrm{Si}-\mathrm{Si}$ dimer distance is found to be $2.45 \AA$, a little larger than what is found on the $\mathrm{Si}(001)$ surface. Because of this dimer formation, the surface becomes $(2 \times 1)$ reconstructed. A top view of this $(2 \times 1)$ reconstructed surface is shown in Fig. 2 . This doubling of unit cell along [011] has been seen in LEED experiments, though this effect was found to be "weak." In addition to forming dimers, the edge $\mathrm{Si}$ atoms move up by $\sim 0.6 \AA$ along the [211] direction relative to the corresponding terrace atoms. In STM experiments by the same authors, the extended ordered atomic rows along [01 $\overline{1}$ ] were found to be made of two asymmetric thin lines close to one another, one being positioned at a slightly greater height compared to the other. Presumably, it is the difference in height between the terrace and edge atoms that was seen in STM. However, the $(2 \times)$ reconstructions along the [111] direction reported in some experiments (the unit-cell length being doubled to $19.82 \AA$ ) cannot appear in our calculations as we include only two atoms along this direction with a unit-cell length of $9.41 \AA$. The dimerization of the edge $\mathrm{Si}$ atoms leads to an energy gain of $2.1 \mathrm{eV}$ per dimer compared to the ideal surface. Various bond lengths and bond angles for the $(2 \times 1)$ reconstructed surface are given in Table III. A comparison with Ref. 12 shows that the Si-Si dimer distance is marginally greater in our calculation. That is why, $\mathrm{T}(4)$ -
TABLE II. Total binding energy $\left(E_{b}\right)$ of the clean $\mathrm{Si}(211)$ surface for different MP $k$ meshes with $E_{\text {cut }}=250 \mathrm{eV}$.

\begin{tabular}{ccc}
\hline \hline$k$ mesh & Irreducible $k$ points & $E_{b}(\mathrm{eV})$ \\
\hline $1 \times 1 \times 1$ & 1 & -323.661536 \\
$2 \times 2 \times 1$ & 4 & -325.044874 \\
$4 \times 4 \times 1$ & 10 & -324.990027 \\
\hline \hline
\end{tabular}

$\mathrm{E}(4)-\mathrm{L}_{3}(4)$ bond angle is a little smaller. It is noted that the $(2 \times 1)$ reconstructed surface is metallic in character. An alternate way of looking at the $\mathrm{Si}(211)$ surface has been suggested in which the edge atoms are eliminated. ${ }^{1,12}$ In fact, there are experiments that report at least parts of the surface without these edge atoms. ${ }^{6}$ This alternate surface allows a rebonding while maintaining the $(1 \times 1)$ symmetry. ${ }^{1,12,17} \mathrm{We}$ have also seen this rebonded $(1 \times 1)$ surface while relaxing the alternate $\mathrm{Si}(211)$ surface without the edge atoms. The terrace atoms on the surface bond with the corresponding atom along $[1 \overline{1} \overline{1}]$ in the third layer. A model for this rebonded surface is shown in Fig. 3. The bond lengths and angles for this surface are given in Table IV. It is instructive to compare the surface energies for these two reconstructions. Surface energy has been defined in the following way by Chadi: ${ }^{1}$

$$
E_{\text {surf }}=E_{\text {tot }}(N)-N E_{0},
$$

where $E_{\text {surf }}$ is the surface energy (generally positive), $E_{\text {tot }}(N)$ is the total energy of a system of $N$ atoms with an exposed surface, and $E_{0}$ is the total energy per $\mathrm{Si}$ atom in the bulk. This is easy to see, as any difference between two systems - one with an exposed surface and the other an infinite bulk-will arise from the surface effects and is a measure of the energy required to create the surface. Grein used this definition to calculate the surface energies of the $(2 \times 1)$ and rebonded $(1 \times 1)$ surfaces in his calculation. ${ }^{12}$ Note that in that calculation, the dangling bonds in the lowermost $\mathrm{Si}$ layer were not saturated by $\mathrm{H}$. However, it was possible to take care of the effects of these dangling bonds while calculating the surface energy of the reconstructed surface at the top. In the present calculations, in contrast, we have saturated the dangling bonds at the bottom by $\mathrm{H}$. This allows one to simulate a bulklike termination at the bottom with a relatively small slab thickness. Unfortunately, this also requires us to know the contribution of the $\mathrm{Si}-\mathrm{H}$ bonds to the total energy of the system if we wish to calculate the surface energies from these calculations. Although we are unable to obtain the surface energies of the $(2 \times 1)$ and rebonded $(1 \times 1)$ surfaces separately, our main interest is to calculate the difference in the surface energies of these two reconstructions. This indeed can be done, since the unknown contribution from the Si-H bonds is exactly the same in the two systems and cancels out exactly. Another point to remember while calculating the surface energy difference between the two reconstructions is that the two systems have different number of atoms - the system with $(2 \times 1)$ reconstructed surface has four surface atoms more than the rebonded $(1 \times 1)$ surface. Assuming that the $(2 \times 1)$ and rebonded $(1 \times 1)$ systems have 

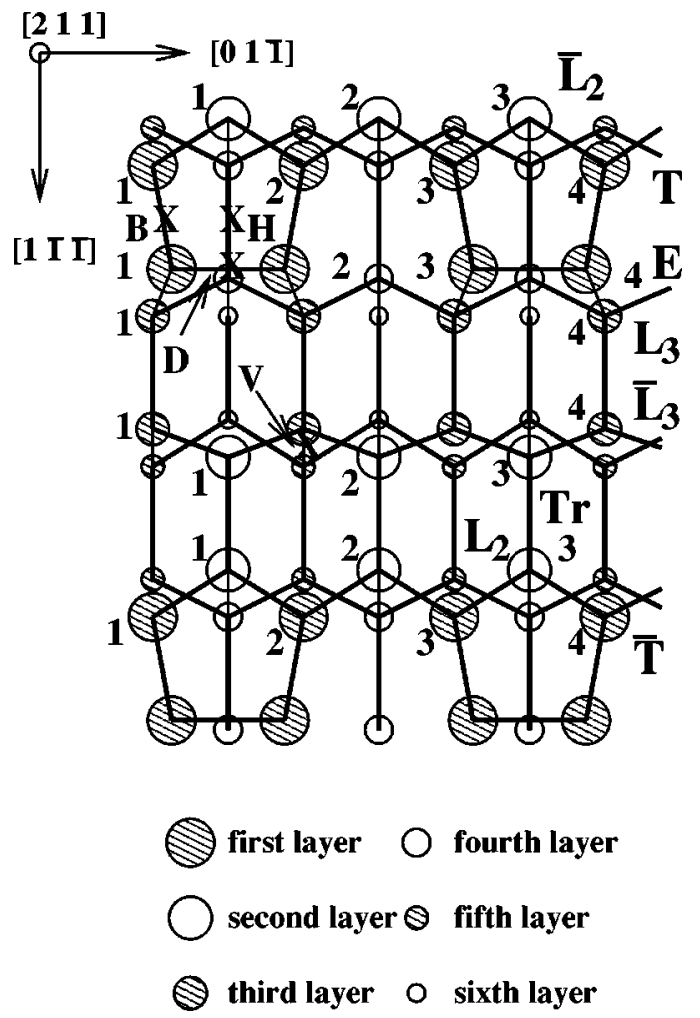

FIG. 2. Top view of the $(2 \times 1)$ reconstructed $\mathrm{Si}(211)$ surface. The twofold coordinated edge atoms dimerize to lower the energy. The four symmetry points on this surface at which adsorption studies have been done are marked. Other rows of atoms in the second and third layers have been marked by $\mathrm{L}_{2}, \overline{\mathrm{L}}_{2}, \mathrm{~L}_{3}$, and $\overline{\mathrm{L}}_{3}$ which need to be identified for noting the bond lengths and bond angles in Table III. Note that $\mathrm{L}_{2}$ and $\overline{\mathrm{L}}_{2}$ rows are symmetry equivalent and so are $\mathrm{T}$ and $\overline{\mathrm{T}}$.

$N$ and $(N-4) \mathrm{Si}$ atoms forming the slabs, respectively, from the above argument we can write

$$
E_{\text {surf }}(2 \times 1)=E_{\text {total }}(2 \times 1, N)-N E_{0}+E_{\mathrm{Si}-\mathrm{H}} \cdot
$$

The surface energy of a rebonded $(1 \times 1)$ surface can be written as

TABLE III. Selected bond lengths and bond angles for a $(2 \times 1)$ surface.

\begin{tabular}{lc}
\hline \hline Atoms & Bond length $(\AA)$ or angle $\left(^{\circ}\right)$ \\
\hline \hline $\mathrm{T}(4)-\mathrm{E}(4)$ & 2.26 \\
$\mathrm{E}(3)-\mathrm{E}(4)$ & 2.45 \\
$\mathrm{E}(4)-\mathrm{L}_{3}(4)$ & 2.37 \\
$\mathrm{~L}_{3}(4)-\overline{\mathrm{L}}_{3}(4)$ & 2.35 \\
$\overline{\mathrm{L}}_{3}(4)-\mathrm{Tr}(3)$ & 2.32 \\
$\operatorname{Tr}(3)-\mathrm{L}_{2}(3)$ & 2.36 \\
$\overline{\mathrm{L}}_{2}(3)-\mathrm{T}(4)-\mathrm{E}(4)$ & 88.67 \\
$\mathrm{~T}(4)-\mathrm{E}(4)-\mathrm{L}_{3}(4)$ & 105.04 \\
$\mathrm{E}(4)-\mathrm{L}_{3}(4)-\overline{\mathrm{L}}_{3}(4)$ & 108.42 \\
\hline \hline
\end{tabular}
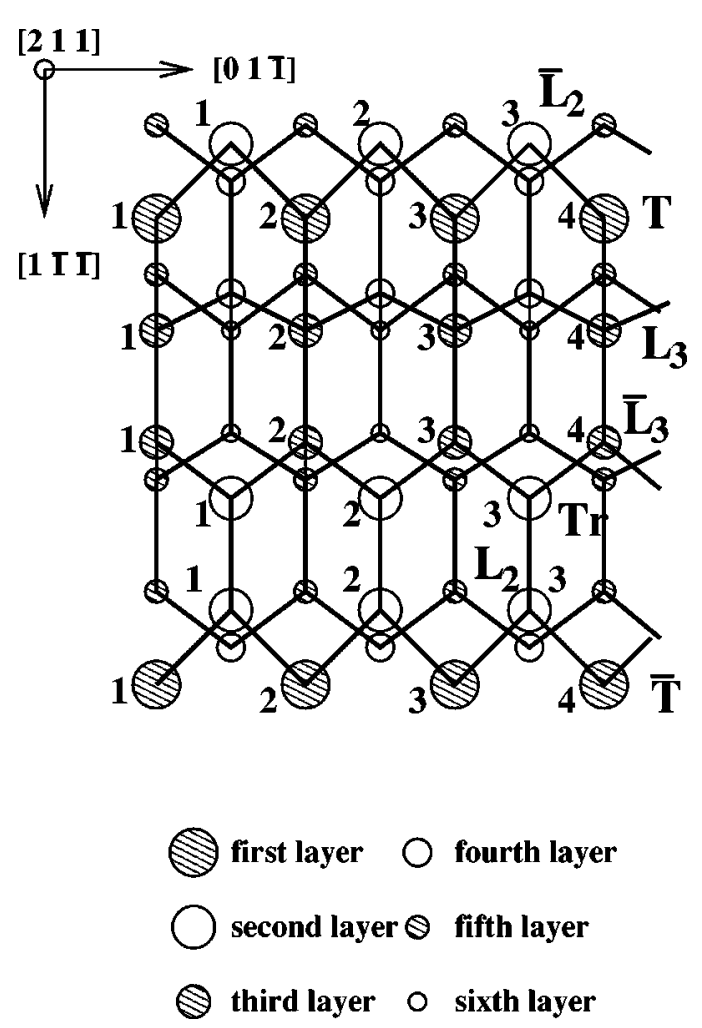

FIG. 3. Top view of a $(1 \times 1)$ rebonded $\operatorname{Si}(211)$ surface. The edge atoms are missing. The top layer atoms bind with the corresponding third-layer atoms retaining the $(1 \times 1)$ symmetry of the surface. The second- and third-layer rows have been identified for the bond lengths and bond angles in Table IV.

$$
E_{\text {surf }}(1 \times 1)=E_{\text {total }}(1 \times 1, N-4)-(N-4) E_{0}+E_{\mathrm{Si}-\mathrm{H}},
$$

where $E_{\mathrm{Si}-\mathrm{H}}$ is the unknown contribution to the energy from the Si-H bonds. Taking the difference of Eqs. (2) and (3), and using the values of the energies from our calculations, we find

$$
E_{\text {surf }}(2 \times 1)-E_{\text {surf }}(1 \times 1)=-1.9356 \mathrm{eV} .
$$

The surface area of the $\mathrm{Si}$ slab in our supercell is $144.914 \AA^{2}$. This gives an energy difference of $0.013 \mathrm{eV} / \AA^{2}$ between the two surfaces. It should be noted here that like in

TABLE IV. Selected bond lengths and bond angles for the $(1 \times 1)$ surface.

\begin{tabular}{lc}
\hline \hline Atoms & Bond length $(\AA)$ and angle $\left(^{\circ}\right)$ \\
\hline $\mathrm{T}(4)-\mathrm{L}_{3}(4)$ & 2.46 \\
$\mathrm{~L}_{3}(4)-\overline{\mathrm{L}}_{3}(4)$ & 2.52 \\
$\overline{\mathrm{L}}_{3}(4)-\mathrm{Tr}(3)$ & 2.34 \\
$\mathrm{Tr}(3)-\mathrm{L}_{2}(3)$ & 2.41 \\
$\mathrm{~L}_{2}(3)-\overline{\mathrm{T}}(4)$ & 2.43 \\
$\mathrm{~L}_{2}(3)-\mathrm{T}(4)-\mathrm{L}_{3}(4)$ & 101.80 \\
$\mathrm{~T}(4)-\mathrm{L}_{3}(4)-\overline{\mathrm{L}}_{3}(4)$ & 137.56 \\
$\mathrm{~L}_{3}(4)-\overline{\mathrm{L}}_{3}(4)-\mathrm{Tr}(3)$ & 120.45 \\
\hline \hline
\end{tabular}


Ref. 12, the energy difference between the two surface reconstructions is rather small and is at the level of accuracy of these calculations. Hence, depending on the actual experimental conditions, one may end up with either surface. Another reconstruction that was proposed as a possibility for the $\mathrm{Si}(211)$ surface is a $\pi$-bonded chain formation as on the $\mathrm{Si}(111)$ surface. A model for $\pi$-bonded chain reconstruction on the $\mathrm{Si}(211)$ surface is shown in Fig. 4, in which the top layer terrace atoms dimerize with the corresponding fourthlayer atoms. ${ }^{18}$ We investigate this possibility extensively. First, we push down the top layer terrace atoms by $\sim 1.1 \AA$ so that they are at the same height as the second-layer atoms to facilitate any possible dimerization, and relax the structure. In the optimized structure, the top layer terrace atoms are pushed back up to their original height and the edge atoms form dimers along the [011] direction, leading to the $(2 \times 1)$ structure. Next, we start from an initial geometry in which the top layer terrace atoms are pushed down substantially so that the distance between them and the corresponding fourth-layer atoms is $\sim 2.3 \AA$, close to the $\mathrm{Si}-\mathrm{Si}$ bond length. Then, as a first step, these atoms forming the "dimer" are fixed along with the two lowermost Si layers and the $\mathrm{H}$ layer, while all the other atoms are relaxed. The energy of this configuration is found to be higher than that in a $(2 \times 1)$ reconstructed surface. Starting with this intermediate geometry, all the atoms in the top five layers are allowed to move (including the dimers that were held fixed) for further structural optimization. The top layer atoms again move up. The surface ends up in the same $(2 \times 1)$ reconstruction with the same energy as obtained starting from an ideal surface. Thus we conclude that the $\pi$-bonded chain is not a stable reconstruction for this surface.

\section{B. Arsenic adsorption}

In this section, we discuss our results of As adsorption on the $\operatorname{Si}(211)$ surface. Though it has been reported that the clean $\mathrm{Si}(211)$ can form a rebonded $(1 \times 1)$ surface where the edge atoms are missing, none of the adsorption experiments on this surface report this. Also, as noted above, our calculations give a slightly lower surface energy for the $(2 \times 1)$ surface. Hence, we have restricted our adsorption studies only to the ideal and the $(2 \times 1)$ reconstructed surfaces.

\section{As on ideal Si(211)}

A stepped surface such as $\mathrm{Si}(211)$ has many inequivalent atoms on the surface and hence offers a number of possible adsorption sites. We study the adsorption of an As atom on six different symmetry sites on the ideal $\mathrm{Si}(211)$ surface. These symmetry points are labeled in Fig. 1. The T, E, and Tr sites are on top of the terrace, edge, and trench $\mathrm{Si}$ atoms marked in the figure. The trench atoms are the second-layer $\mathrm{Si}$ atoms that are also threefold bonded. The bridge site (B) is on top of the bond between the surface terrace and edge atoms, while the hollow site $(\mathrm{H})$ is in the middle of the rectangle on the surface formed by two terrace and the two corresponding edge atoms. The valley site $(\mathrm{V})$ is halfway between two second-layer trench atoms. We have selected a set of plausible adsorption sites from our intuitive notions of

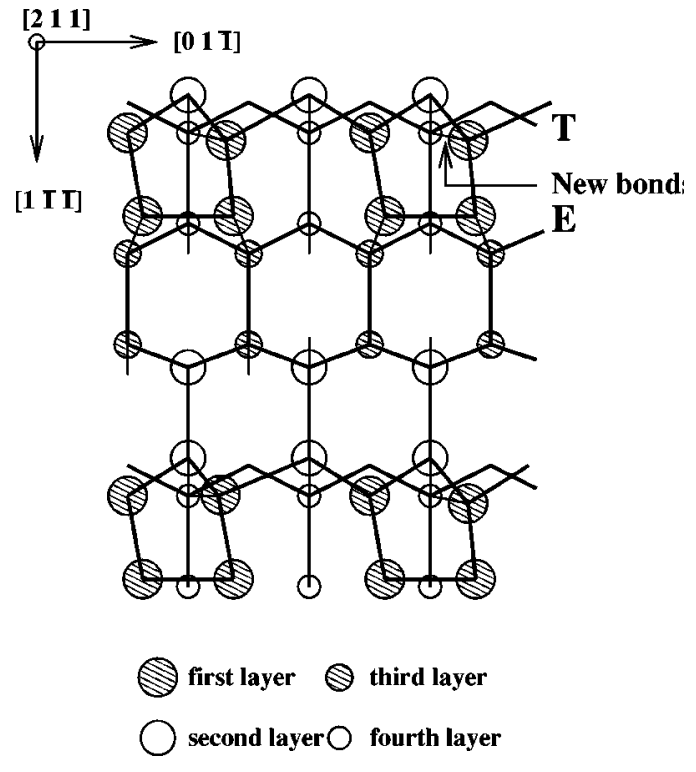

FIG. 4. The proposed $\pi$-bonded chain reconstruction of $\operatorname{Si}(211)$ surface. The top layer $\mathrm{T}$ atoms bind with the corresponding fourthlayer atoms. Only the top four-layers have been shown for clarity.

chemical bonding. The binding energies of an As atom at various sites and its distance from the nearest $\mathrm{Si}$ atoms(s) are given in Table $\mathrm{V}$. The $\mathrm{B}$ site turns out to be energetically the most favorable one for As adsorption. This is followed by the $\mathrm{H}, \mathrm{V}, \mathrm{E}, \mathrm{Tr}$, and $\mathrm{T}$ sites. This sequence of binding energies can be rationalized in the following way. At the B site, the As can bind to the terrace and edge $\mathrm{Si}$. With the edge $\mathrm{Si}$, which has two dangling bonds left, it can form a double bond. The asymmetry in the binding of the As with two surface $\mathrm{Si}$ atoms at the $\mathrm{T}$ and $\mathrm{B}$ sites is clearly seen in the chargedensity plot in Fig. 5. This satisfies the dangling bonds of the terrace and edge Si atoms. The As is threefold bonded and its remaining two electrons can from a lone pair. At the $\mathrm{H}$ site, the As forms four weak bonds with the four neighboring $\mathrm{Si}$ atoms - two terrace and two edge - as can be seen from the larger $\mathrm{Si}$-As bond lengths. However, formation of four bonds reduces the number of dangling bonds. Arsenic is left with one dangling bond and the edge $\mathrm{Si}$ atoms with one dangling bond each. The terrace Si's have their bonds satisfied. This reduction of dangling bonds leads to quite large binding energy at this site. On top of the edge, terrace and trench atoms, the As can bind with only one Si atom. Thus the binding energies are unfavorable at these sites. Out of these three, the binding energy at the E site is the highest. This is presumably because As forms a double bond with the edge Si whereby satisfying its dangling bonds and two of its own. At the T and $\mathrm{Tr}$ sites, however, it can form only one bond with the corresponding $\mathrm{Si}$ atoms as they have only one dangling bond each. This conjecture is supported by the fact that at the E site, the As-Si bond length is the shortest indicating a stronger binding. On the other hand, at the V site, the As can form bonds with two neighboring trench $\mathrm{Si}$ atoms, which have their remaining dangling bonds satisfied in the process. This makes the binding energy at the $\mathrm{V}$ site comparable to that at the $\mathrm{B}$ and $\mathrm{H}$ sites. 
TABLE V. Distances of the As adatom at various symmetry points on the surface from the neighboring $\mathrm{Si}$ atoms of an ideal surface. The corresponding binding energies are also given.

\begin{tabular}{|c|c|c|c|c|c|c|c|}
\hline \multicolumn{2}{|l|}{ As } & \multicolumn{4}{|c|}{ Distance $(\AA)$ from which $\mathrm{Si}$} & \multirow[b]{2}{*}{$\operatorname{Tr}(2)$} & \multirow[b]{2}{*}{$E_{b}(\mathrm{eV})$} \\
\hline Position & $\mathrm{E}(1)$ & $\mathrm{E}(2)$ & $\mathrm{T}(1)$ & $\mathrm{T}(2)$ & $\operatorname{Tr}(1)$ & & \\
\hline B & 2.30 & & 2.30 & & & & 5.37 \\
\hline $\mathrm{H}$ & 2.61 & 2.61 & 2.61 & 2.61 & & & 5.30 \\
\hline V & & & & & 2.59 & 2.59 & 5.05 \\
\hline $\mathrm{E}$ & 2.16 & & & & & & 4.55 \\
\hline $\mathrm{T}$ & & & 2.24 & & & & 3.85 \\
\hline $\operatorname{Tr}$ & & & & & 2.26 & & 4.28 \\
\hline
\end{tabular}

\section{As on $\operatorname{Si}(211)-(2 \times 1)$}

We now discuss our studies of As adsorption on the $(2 \times 1)$ reconstructed $\mathrm{Si}(211)$ surface. We have already seen in the case of the ideal surface that the sites on top of various surface atoms, where the As binds to only one $\mathrm{Si}$ atom, are not favorable energetically. So we do not consider those sites anymore. We study the adsorption of As on the B, V, and $\mathrm{H}$ sites. In addition, we consider As adsorption on top of the surface Si-Si dimer (D site, see Fig. 2). For these calculations, the planar position of the As adatom is held fixed while it is allowed to relax its height. The top five Si layers are fully relaxed as before. On the $(2 \times 1)$ reconstructed surface, the $\mathrm{V}$ site turns out to be the most favorable one for an As adatom with a binding energy $E_{b}^{V}=4.84 \mathrm{eV}$. This is followed by the $\mathrm{B}, \mathrm{D}$, and $\mathrm{H}$ sites with $E_{b}^{B}=4.69 \mathrm{eV}, E_{b}^{D}=4.32 \mathrm{eV}$, and $E_{b}^{H}=3.81 \mathrm{eV}$. The geometries of the surface around the As adatom in these four cases are shown in Fig. 6 . At the $\mathrm{V}$ site, As binds with the two neighboring second-layer Si atoms. Similarity of As adsorption at this site to that on the $\mathrm{Si}(001)$ surface is noticeable. A monolayer of As adsorbed on $\mathrm{Si}(001)$ has As-Si bond length equal to $2.44 \AA .{ }^{19}$ As adsorbed on the $\mathrm{V}$ site has the same As-Si bond length. At the B site,

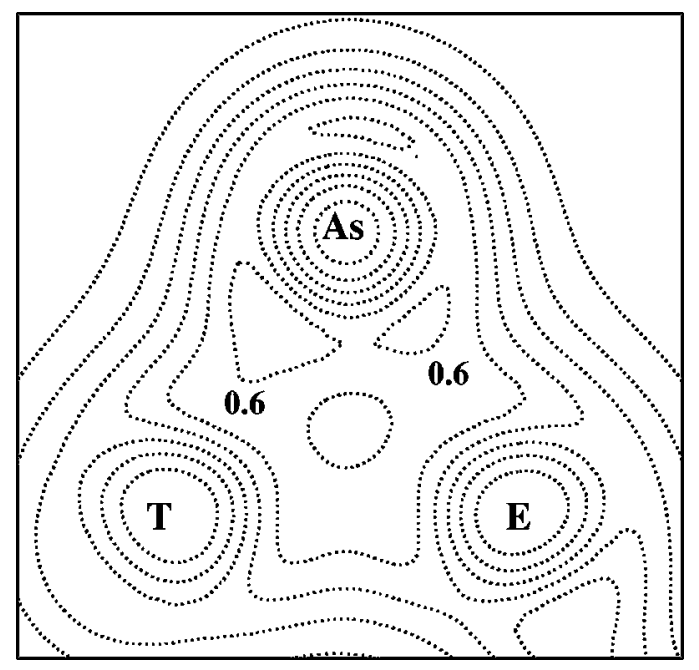

FIG. 5. Charge-density contour plot in the Si-As-Si plane ( $y-z$ plane in our calculation) for an isolated As adatom at the B site of an ideal $\mathrm{Si}(211)$ surface. The asymmetry in bonding of the As with the terrace and edge $\mathrm{Si}$ atoms is apparent.

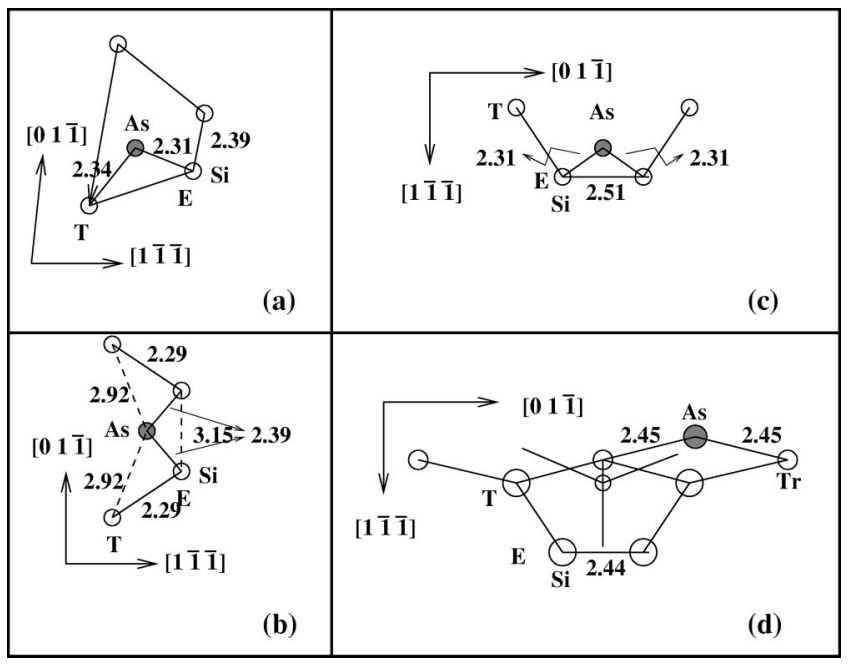

FIG. 6. The local geometry around the adsorbed As atom at the B (a), H (b), D (c), and V (d) sites of the $(2 \times 1)$ reconstructed $\mathrm{Si}(211)$ surface. Please note that the direction of view is slightly different in different cases. All the distances are given in angstrom.

the As binds to two surface Si atoms as on an ideal surface. However, it cannot from a double bond with the edge $\mathrm{Si}$ as on an ideal surface, since the edge $\mathrm{Si}$ is already threefold coordinated because of the $\mathrm{Si}-\mathrm{Si}$ dimerization. Binding with the As satisfies the dangling bonds of the terrace and edge $\mathrm{Si}$ atoms. In spite of having the same dangling-bond density, As adsorbed at the $\mathrm{V}$ site has a higher binding energy probably because of the optimal length of the As-Si bond, which is much shorter $(\sim 2.3 \AA)$ in case of As on the B site. Interestingly, the presence of the As adatom at the B site reduces the Si-Si dimer distance marginally to $2.39 \AA$. At the D site, the As binds with the two edge $\mathrm{Si}$ atoms forming the dimer. However, the presence of the As atom in this case increases the $\mathrm{Si}$-Si dimer distance marginally to $2.52 \AA$, costing energy. This makes the binding energy on this site slightly lower than that at the B site. At the $\mathrm{H}$ site, As prefers to form bonds of optimal length with the two edge Si atoms and does not bind with the terrace atoms as can be seen from the interatomic distances shown in Fig. 6. Apparently, this cannot be achieved by keeping all the $\mathrm{Si}-\mathrm{Si}$ bonds intact. Consequently, the $\mathrm{Si}-\mathrm{Si}$ dimer bond is broken making the $\mathrm{Si}-\mathrm{Si}$ distance $3.15 \AA$ and costing the $\mathrm{Si}-\mathrm{Si}$ binding energy. This is in contrast to the behavior of $(2 \times 1)$ reconstructed $\mathrm{Si}(001)$ surface on which As and Te are known to break the Si-Si surface dimers adsorbing on top of the dimers. This breaking of the Si-Si dimers makes the binding at this site the least beneficial energetically. However, it must be noted that though the binding energy at the hollow site is quite low on the $(2 \times 1)$ surface compared to an ideal one, the total energy is still lower in the former case. We have also studied the effects of a higher coverage of As on the $(2 \times 1)$ reconstructed $\mathrm{Si}(211)$ surface. Although the $\mathrm{V}$ site turned out to be the most favorable one for an isolated As adsorption, we put the As atoms at the next most favorable site, namely the B site, at higher coverages. This is because, at the V site, the As adatom does not have any major effect on the geometry of the surface apart from binding with the two neighboring $\mathrm{Tr}$ at- 

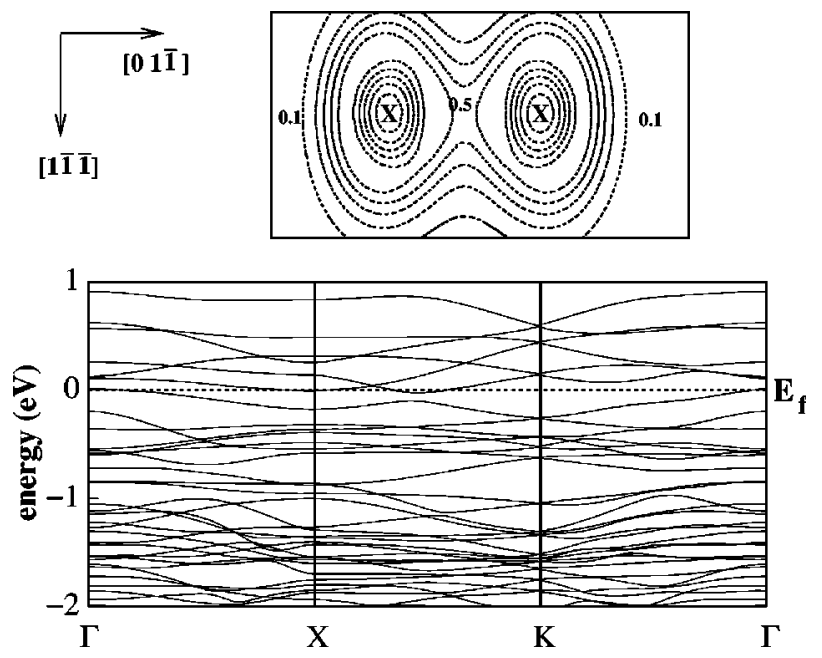

FIG. 7. The top panel shows the charge-density plot in the plane of the As atoms for 0.5 -ML coverage. The maximum charge density halfway between the two As atoms at the B sites indicates dimerization. The bottom panel shows the band structure of the same system.

oms. So, a priori it seems that at higher coverage, As atoms, if adsorb at the alternate $\mathrm{V}$ sites, would bind with the $\mathrm{Tr}$ atoms without causing any change in the reconstruction of the surface. Therefore, at a coverage of $0.5 \mathrm{ML}$ (monolayer) that we study, we put four As atoms on the four B sites of the $(4 \times 2)$ surface supercell that we use. At 0.5 -ML coverage, we find a binding energy of $5.28 \mathrm{eV}$ per As adatom. This is larger than the binding energy of a single As adatom on a B site. With As adatoms on all the $\mathrm{B}$ sites, the $\mathrm{Si}-\mathrm{Si}$ dimer distances decrease to $2.31 \AA$. This effect was already seen in the presence of a single As atom at a B site. The presence of more As adatoms enhances this effect further. Energy is gained because of stronger binding of the surface Si atoms. Further energy is gained due to dimerization of the As adatoms. That the As adatoms indeed dimerize at $0.5 \mathrm{ML}$ can be seen from the charge-density plot in the plane of the As atoms shown in Fig. 7. This figure also shows the band structure of the system along directions parallel to the surface. The system is seen to be metallic in character. In the final optimized structure, the Si-As and As-As distances are 2.44 $\AA$ and $2.55 \AA$, respectively, exactly equal to the corresponding numbers for As adsorption on the $\mathrm{Si}(001)$ surface. The

TABLE VI. Distances of the Te adatom at various symmetry points on the surface from the neighboring $\mathrm{Si}$ atoms of an ideal surface along with the corresponding binding energies.

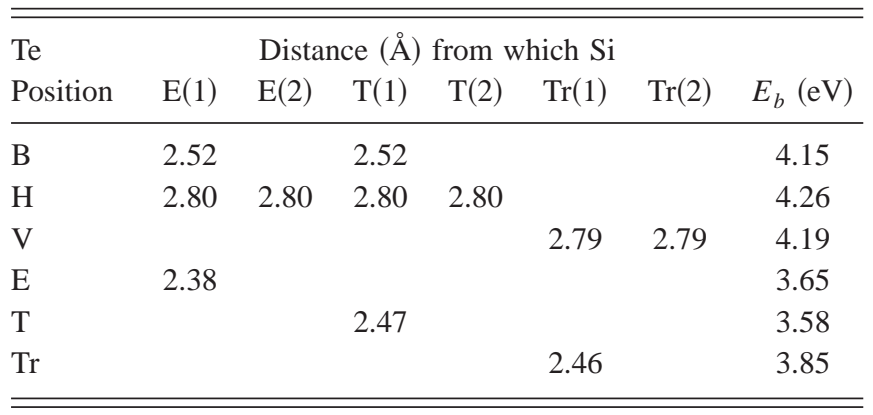

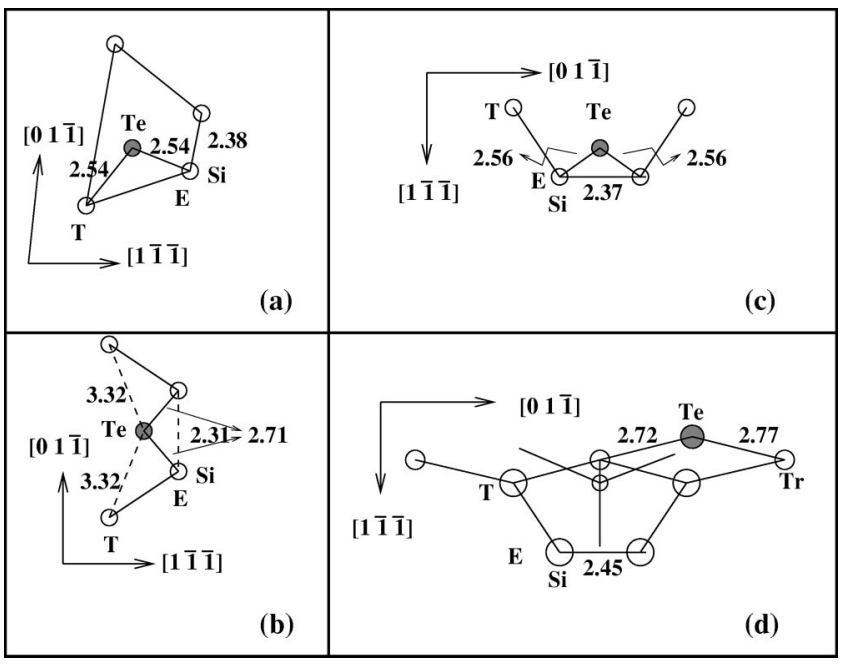

FIG. 8. Local geometry around the Te adatom at various sites on a $(2 \times 1)$ reconstructed $\mathrm{Si}(211)$ surface: (a) B, (b) H, (c) D, and (d) V sites.

surface still retains the $(2 \times 1)$ reconstruction at this coverage, but the origin is due to the dimerization of the As adatoms.

\section{Te adsorption on $\mathrm{Si}(211)$}

In this section, we discuss our studies of Te adsorption on the $\mathrm{Si}(211)$ surface. As in the case of As adsorption, we study adsorption of Te on the ideal and the $(2 \times 1)$ reconstructed surfaces.

\section{Te on ideal $\mathrm{Si}(211)$}

As in the case of As adsorption, we have studied Te adsorption at six special symmetry points on the ideal $\mathrm{Si}(211)$ surface. These sites can be grouped into two categories in terms of the nature of binding and the binding energies. Three sites, namely the H, V, and B sites, have high binding energies which are close to each other. The T, E, and Tr sites,
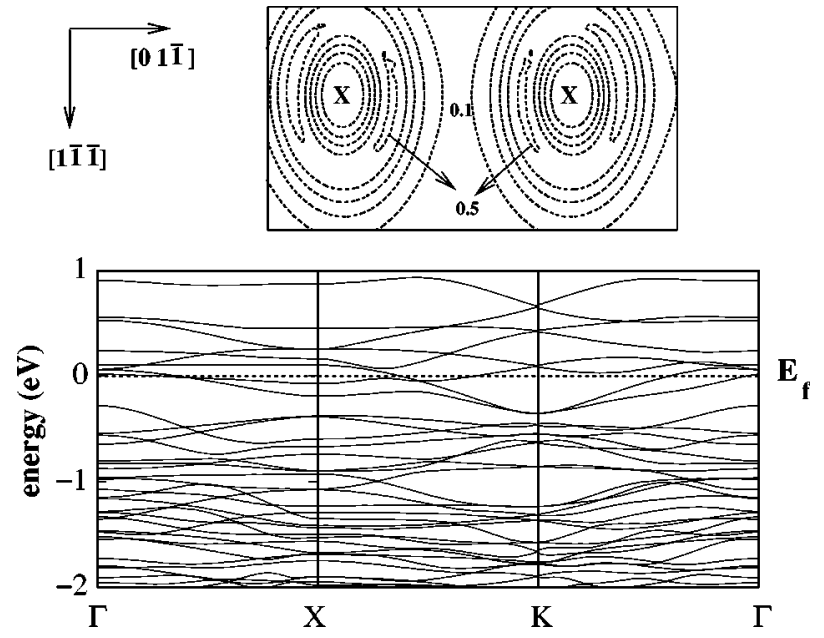

FIG. 9. The top panel shows the charge-density contour plot in the plane of Te atoms for $0.5-\mathrm{ML}$ coverage. Clearly, there is no bonding between the two Te atoms at the $\mathrm{B}$ sites. The bottom panel is the band structure of the same system. 
on the other hand, have relatively low binding energies. The reason is similar to the case of As. At the $\mathrm{H}, \mathrm{V}$, and B sites, the Te binds to four, two and two, $\mathrm{Si}$ atoms, respectively. This reduces the number of dangling bonds leading to high binding energies. Why the binding energies at the $\mathrm{B}$ and $\mathrm{V}$ sites are lower than that at the $\mathrm{H}$ site is not obvious. It appears that the bond-bending energy of the edge $\mathrm{Si}$ atom for a Te at B is rather high. For an As at B, further energy can be gained by forming a double bond with the edge atom which is not possible for a Te adatom. It should also be mentioned that the binding energies at the $\mathrm{V}$ and $\mathrm{B}$ sites are very close and their difference is within the error margins of our calculation. This is in agreement with the experimental observation of Dhar et al. ${ }^{11}$ mentioned before. However, they have no precise information of the binding sites because of the nature of their experiments. For a Te on top of the T, E, and $\mathrm{Tr} \mathrm{Si}$ atoms, the Te adatom can bind to only one $\mathrm{Si}$ atom and thereby have low binding energies. The binding energies and the distances from the nearest $\mathrm{Si}$ atoms are given in Table VI.

\section{Te on $\mathrm{Si}(211)-(2 \times 1)$}

Now we discuss our studies of Te adsorption on the $(2 \times 1)$ reconstructed $\mathrm{Si}(211)$ surface. Again, we study adsorption only on the four sites where the Te adatom can bind to more than one $\mathrm{Si}$ atoms, i.e., the $\mathrm{B}, \mathrm{H}, \mathrm{V}$, and $\mathrm{D}$ sites. The $\mathrm{B}$ site is found to be energetically the most favorable one for Te adsorption with a binding energy of $E_{b}^{B}=4.09 \mathrm{eV}$, which is closely followed by the $\mathrm{V}$ site with $E_{b}^{V}=4.02 \mathrm{eV}$. The $\mathrm{D}$ and $\mathrm{H}$ sites have relatively lower binding energies with $E_{b}^{D}$ $=3.32 \mathrm{eV}$ and $E_{b}^{H}=2.62 \mathrm{eV}$, respectively. It should be mentioned here that the maximum binding-energy values for $\mathrm{Te}$ on $\mathrm{Si}(211)$ found in our calculation are slightly different from that obtained by Dhar et al. ${ }^{11}$ However, that is not surprising given the fact that ours is a complete quantummechanical calculation while their estimate is based on purely classical considerations. Unlike in the case of As, the $\mathrm{Si}-\mathrm{Si}$ dimer is not broken for a Te adsorption on the $\mathrm{H}$ site. If the As-Si bond is stronger than the $\mathrm{Si}-\mathrm{Si}$ bond, the surface $\mathrm{Si}$ dimers can break in favor of the $\mathrm{Si}$ atoms forming double bonds with the adsorbed As thereby gaining energy. This is not possible in the case of Te which has a valence of 2 and hence the Si dimer remains intact. The geometries around the adsorbed Te atom in these four cases are shown in Fig. 8. We have also studied 0.5 -ML Te adsorption on the $(2 \times 1)$ reconstructed $\mathrm{Si}(211)$ surface. Since the B site is the most favorable for Te adsorption, it is reasonable to put the additional Te atoms on the B sites as well. Hence, we have studied the effects of four Te adatoms at the four B sites in our $(4 \times 2)$ surface supercell just as in the case of As. In marked contrast to As adsorption, the Te atoms do not dimerize as can be seen from the charge-density contour plots in the plane of Te atoms shown in Fig. 9. This figure also shows the band structure of the 0.5-ML Te-covered surface. As can be seen, this system is also metallic in character. The binding energy of $4.09 \mathrm{eV}$ per Te adatom is almost the same as for a single Te adatom $(4.08 \mathrm{eV})$. This is consistent with the fact that there is no additional energy gain, unlike the case of As, from adatom dimerization. Moreover, it is also consistent with the chemical notion that $\mathrm{Te}$, having the outermost electronic configuration of $5 s^{2} 5 p^{4}$, is divalent and cannot dimerize after binding with two neighboring $\mathrm{Si}$ atoms. This absence of $\mathrm{Te}-\mathrm{Te}$ dimerization is exactly similar to the case of 1-ML Te adsorption on the $\mathrm{Si}(001)$ surface. ${ }^{20}$ However, in case of the $\mathrm{Si}(211)$ surface, the $(2 \times 1)$ reconstruction of the substrate is retained because the edge $\mathrm{Si}$ atoms retain their dimerization. In the optimized structure, the $\mathrm{Si}-\mathrm{Si}$ dimer distance on the surface is found to be $2.35 \AA$; and the $\mathrm{Si}-\mathrm{Te}$ distance is 2.56 $\AA$. The Si-Te distance is very close to the sum of the atomic radii of $\mathrm{Si}(\sim 1.17 \AA)$ and $\mathrm{Te}(\sim 1.32 \AA)$ and the value for the same quantity found for Te adsorption on the $\mathrm{Si}(001)$ surface. Note that we have not tried to study coverages beyond $0.5 \mathrm{ML}$ because of the additional complication of subsurface adsorption for coverages greater than 0.6 ML, as seen in Ref. 11.

\section{CONCLUSION}

We have performed an extensive plane-wave, pseudopotential density-functional calculations for the electronic and structural properties of the clean and As- and Te-covered $\mathrm{Si}(211)$ surface. The clean surface readily forms a $(2 \times 1)$ reconstruction through dimerization of the edge atoms along the $[01 \overline{1}]$ direction. A rebonded $(1 \times 1)$ surface is also found, but the $\pi$-bonded chain is not a stable reconstruction for this surface. The twofold bridge site is found to be the most favorable one for an isolated As adatom, whereas the fourfold hollow site is the most favorable one for a Te on an ideal $\mathrm{Si}(211)$ surface. On the $(2 \times 1)$ reconstructed surface, the valley site is the most favorable one for As while the bridge site is the most favorable one for an isolated Te. Just as suggested by the experiments on $\mathrm{Br}$ and $\mathrm{Te}$ adsorption on the $\mathrm{Si}(211)$ surface, there are more than one points on the surface with very close binding-energy values for both As and Te. At 0.5 -ML coverage, when there is a line of adatoms along [011] at the bridge sites, the As adatoms dimerize on the $\mathrm{Si}(211)$ surface while Te adatoms do not. In this respect, their behavior is very similar to that on the $\mathrm{Si}(001)$ surface. Both 0.5 -ML As- and Te-covered surfaces are found to be metallic in character.
${ }^{1}$ D.J. Chadi, Phys. Rev. B 29, 785 (1984).

${ }^{2}$ B.Z. Olshanetsky and V.I. Mashanov, Surf. Sci. 111, 414 (1981).

${ }^{3}$ S.L. Wright, H. Kroemer, and M. Inada, J. Appl. Phys. 55, 2916 (1984).

\footnotetext{
${ }^{4}$ S. Rujirawat, L.A. Almeida, Y.P. Chen, S. Sivananthan, and D.J. Smith, Appl. Phys. Lett. 71, 1810 (1997).

${ }^{5}$ B. Yang, Y. Xin, S. Rujirawat, N.D. Browning, and S. Sivananthan, J. Appl. Phys. 88, 115 (2000).
} 
${ }^{6}$ T. Berghaus, A. Brodee, H. Neddermeyer, and S. Tosch, Surf. Sci. 184, 273 (1987).

${ }^{7}$ X. Wang and W.H. Weinberg, Surf. Sci. 314, 71 (1994).

${ }^{8}$ R. Kaplan, Surf. Sci. 116, 104 (1982).

${ }^{9}$ Y. Yang and E.D. Williams, Surf. Sci. 215, 102 (1989).

${ }^{10}$ E.G. Michel, V. Eteläniemi, and G. Materlik, Surf. Sci. 269, 89 (1992).

${ }^{11}$ N.K. Dhar, N. Goldsman, and C.E.C. Wood, Phys. Rev. B 61, 8256 (2000).

${ }^{12}$ C.H. Grein, J. Cryst. Growth 180, 54 (1997).

${ }^{13}$ S. Mankefors, Surf. Sci. 443, 99 (1999).

${ }^{14}$ D. Vanderbilt, Phys. Rev. B 41, 7892 (1990); G. Kresse and J. Hafner, J. Phys.: Condens. Matter 6, 8245 (1994).
${ }^{15}$ J.P. Perdew and Y. Wang, Phys. Rev. B 46, 6671 (1992).

${ }^{16}$ G. Kresse and J. Hafner, Phys. Rev. B 47, R558 (1993); G. Kresse and J. Furtmüller, ibid. 54, 11169 (1996).

${ }^{17}$ K.C. Pandey, in Proceedings of the 16th International Conference on the Physics of Semiconductors, edited by M. Averous (NorthHolland, Amsterdam, 1983), p. 761.

${ }^{18}$ K.C. Pandey, Phys. Rev. Lett. 47, 1913 (1981); J.E. Northrup and M.L. Cohen, ibid. 49, 1349 (1982).

${ }^{19}$ R.I.G. Uhrberg, R.D. Bringans, R.Z. Bachrach, and J.E. Northrup, Phys. Rev. Lett. 56, 520 (1986).

${ }^{20}$ P. Sen, S. Ciraci, I.P. Batra, C.H. Grein, and S. Sivananthan, Surf. Sci. 519, 79 (2002). 